\title{
智能薄膜材料的“折纸术”
}

李星 ${ }^{1}$, 汪洋 ${ }^{1}$, 胥博瑞 ${ }^{1}$, 田子傲 ${ }^{2 *}$, 狄增峰 ${ }^{2}$, 梅永丰 ${ }^{*}$

1. 复旦大学材料科学系, 上海 200433 ;

2. 中国科学院上海微系统与信息技术研究所, 信息功能材料国家重点实验室, 上海 200050

* 联系人, E-mail: zatian@mail.sim.ac.cn; yfm@fudan.edu.cn

\section{Rolling origami with smart materials}

\author{
Xing $\mathrm{Li}^{1}$, Yang Wang ${ }^{1}$, Borui $\mathrm{Xu}^{1}$, Ziao Tian ${ }^{2 *}$, Zengfeng $\mathrm{Di}^{2} \&$ Yongfeng $\mathrm{Mei}^{{ }^{*}}$ \\ ${ }^{1}$ Department of Materials Science, Fudan University, Shanghai 200433, China; \\ ${ }^{2}$ State Key Laboratory of Functional Materials for Informatics, Shanghai Institute of Microsystem and Information Technology, Chinese Academy of \\ Sciences, Shanghai 200050, China \\ * Corresponding authors, E-mail: zatian@mail.sim.ac.cn; yfm@fudan.edu.cn
}

doi: 10.1360/TB-2019-0051

智能材料是一种感知外界环境变化并经过自身判断 后改变特性从而适应这种变化的新型功能材料, 其自变 形、自判断和自适应等特点可以实现对生物智能的人工模 仿, 因而在各领域都有着重要的应用. 在实际应用中, 智 能薄膜材料的 “智能” 程度往往会受限于它的二维尺度, 如 应激行为单一、特性变化测试困难等. 但智能材料的三维 介观结构会表现与平面材料所不同的特性从而实现更好 的智能化. 这些不同的特性如产生负折射率、精确控制电

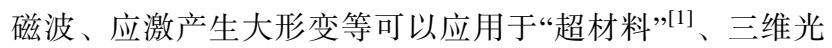
电探测器 ${ }^{[2]}$ 和柔性器件 ${ }^{[3]}$ 等领域. 因此, 实现平面薄膜材 料的三维构筑成为研究的热点. 然而, 这样的结构转变在 细微观下存在着几个难点: 小型化、高集成度和可设计性 等. 针对这些难点, 研究者提出了一种基于应力控制的折 叠方法, 充分利用了智能纳米薄膜的制备工艺. 该方法利 用预应变工程将卷曲纳米技术与精细加工技术结合, 实现 了薄膜的精密图案化, 探索了可拓展的、完全集成的、适 用于各类材料的介观结构制备 ${ }^{[4,5]}$, 我们称这样的方法为 “折纸术”. 该方法同时还具有极强的材料兼容性, 可以结 合包括聚合物、金属以及氧化物在内的 ${ }^{[6,7]}$ 多类别材料. 由 于卷曲纳米薄膜较高的能量密度, 对应变的敏感性和薄膜 自身的柔软性, 智能薄膜材料“折纸”后的三维卷曲结构在 刺激下具有更快的响应速度和更大的形变量, 从而使智能 三维结构器件具有更广阔的应用前景.

本研究团队通过将“折纸术”与钯、二氧化钒等智能材 料薄膜相结合, 成功在片上实现了高集成度、高灵敏度及 大形变的三维卷曲结构功能器件的制备, 并应用于探测器 与制动器等领域. 相关成果发表在 Science Advances ${ }^{[8]}$, Nano Letter ${ }^{[9]}$.
我们设计了一种基于钯 $(\mathrm{Pd})$ 卷曲结构的氢探测器, 其 原理是钯在氢气环境中会受刺激产生一定的响应行为 ${ }^{[8]}$. 将图形化的纳米薄膜逐层沉积到光刻胶上以构建预应力, 在该预应力释放后薄膜卷曲形成大规模的三维结构阵列。 由于吸收氢气后 Pd的体积会膨胀, 当该探测器置于氢气中 时, 整个卷曲结构阵列会从管状结构均匀地转变为平面状 态, 如图 1(a)所示. 同时, 由于纳米薄膜对体积膨胀产生的 应变具有高度敏感性, 该卷曲结构在受到刺激后的响应时 间为 $3.4 \mathrm{~s}$, 恢复时间为 $7.6 \mathrm{~s}$. 基于以上两种性能, 我们开 发了一种可视化探测器. 如图 1(b)和(c)所示, “FUDAN”的 字符和标志在有无氢气的情况下产生了巨大的视觉差 异, 这是因为探测器上卷曲结构的大面积阵列和极快的 响应时间, 使氢气刺激下产生的结构变化在宏观上是可 见的.

我们还设计了一种基于二氧化钒 $\left(\mathrm{VO}_{2}\right)$ 卷曲结构的微 致动器 ${ }^{[9]} . \mathrm{VO}_{2}$ 是一种在 $68^{\circ} \mathrm{C}$ 发生金属-绝缘体转变(MIT) 的相变材料, 相变时会伴随一定的应变变化. 我们通过 $\mathrm{Cr} / \mathrm{VO}_{2}$ 双层结构实现纳米薄膜的卷曲. 研究发现, 随着 $\mathrm{Cr}$ 层厚度的不同, 纳米薄膜的内应变也会改变, 从而制备了 不同曲率的卷曲结构, 如图 1(d)所示. 有意思的是, 在加 热-冷却过程中制动的曲率变化发现, 不同初始曲率的卷 曲结构表现出不同的制动触发温度. 此外, 图 1(e)展示了 该卷曲结构的制动方式: 升温会使卷曲结构转变为平面薄 膜; 而降温则使平面薄膜重新卷曲成管状结构. 这样的大 形变使该制动器的制动效率远高于传统制动器, 这是因为 纳米薄膜的高能量密度, 导致小的应变变化 $(<1 \%)$ 也能实 现大的形变. 在制动原理相似的微纳机器人领域, 通过“折 纸术”实现智能材料上可编程的三维结构设计 ${ }^{[10]}$, 所制备 

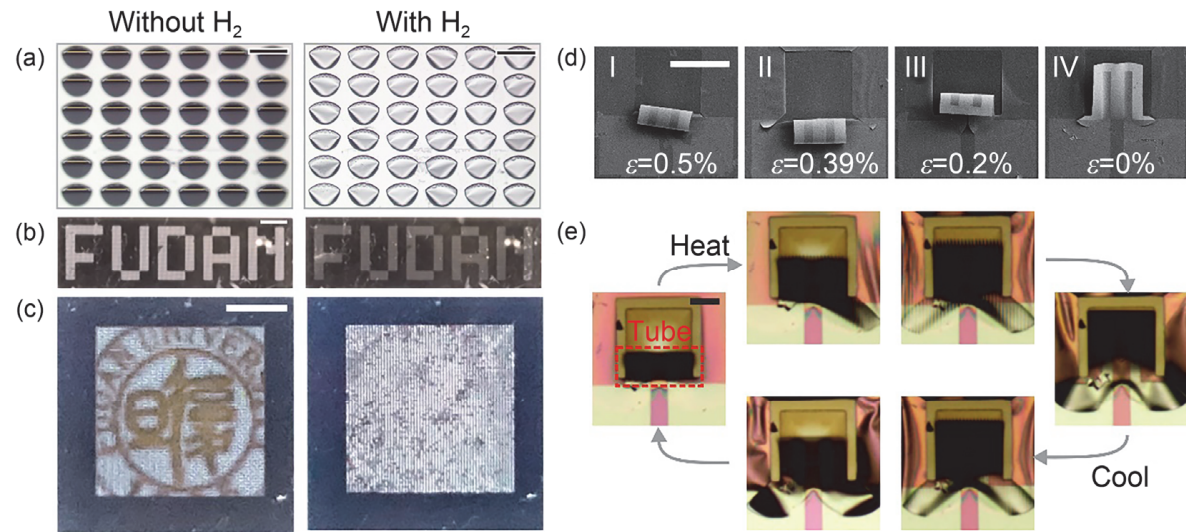

(e)
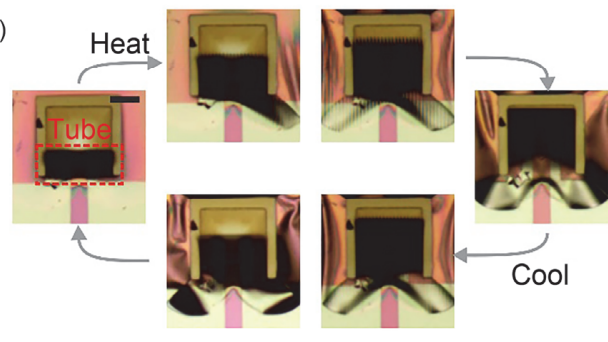

图 1 (网络版彩色)基于智能材料的卷曲微结构 ${ }^{[8,9]}$. (a) 基于卷曲结构的氢探测器在有无氢气情况下的结构变化图. 比例尺: $500 \mu \mathrm{m}$; (b) 通过“FUDAN”字符增强了氢探测在有无氢气情况下的可视度. 比例尺：2 mm; (c) 通过“FUDAN”标志增强氢探测在有无氢气情况下的 可视度. 比例尺: $2 \mathrm{~mm}$; (d) 基于不同曲率卷曲结构的温度激发制动器. 比例尺: $100 \mu \mathrm{m}$; (e) 卷曲微制动器在加热-冷却过程中的曲率变 化. 比例尺: $50 \mu \mathrm{m}$

Figure 1 (Color online) Rolled-up microstructures with smart materials ${ }^{[8,9]}$. (a) Scheme of hydrogen detection and high integration devices based on rolled-up microstructures. Scale bars, $500 \mu \mathrm{m}$. (b) Enhanced macroscopic visual hydrogen detection with "FUDAN" character. Scale bar, $2 \mathrm{~mm}$. (c) Enhanced macroscopic visual hydrogen detection with "FUDAN" logo. Scale bar, $2 \mathrm{~mm}$. (d) Scheme of temperature-dependent microactuators and controllable rolled-up structures with different curvatures. Scale bar, $100 \mu \mathrm{m}$. (e) The curvature changes of rolled-up microactuators during heating and cooling. Scale bar, $50 \mu \mathrm{m}$

的不同结构机器人也具有上述功能器件的优异特征, 如极 快的响应速度与大形变, 为实现更“智能”的机器人提供了 巨大的帮助.

由此可见，“折纸术”可以通过精确的设计将平面智能 材料转变为可预见的三维微结构功能器件, 并使该器件具
有高集成度、出色的应变敏感性和极佳的柔软性. 这些特 性使基于超薄纳米薄膜的智能器件在同类器件中具有非 常明显的优势. 因此, 我们相信在“折纸术”与智能材料的 搭配下, 三维功能器件将应用于包括三维探测器、可穿戴 设备和微纳机器人等更广阔的领域中.

\section{推葆阅读文献}

1 Shelby R A, Smith D R, Schultz S. Experimental verification of a negative index of refraction. Science, 2001, 292: 77-79

2 Lin C H, Tsai D S, Wei T C, et al. Highly deformable origami paper photodetector arrays. ACS Nano, 2017, 11: 10230-10235

3 Song Z, Ma T, Tang R, et al. Origami lithium-ion batteries. Nat Commun, 2014, 5: 3140

4 Rogers J, Huang Y G, Schmidt O G, et al. Origami MEMS and NEMS. MRS Bull, 2016, 41: 123-129

5 Ning X, Wang X J, Zhang Y, et al. Assembly of advanced materials into 3D functional structures by methods inspired by origami and kirigami: A review. Adv Mater Interfaces, 2018, 5: 1800284

6 Xu B R, Zhang B R, Wang L, et al. Tubular micro/nanomachines: From the basics to recent advances. Adv Funct Mater, 2018, 28: 1705872

7 Tian Z A, Zhang L N, Fang Y F, et al. Deterministic self-rolling of ultra-thin nanocrystalline diamond nanomembranes for three-dimensional tubular/helical architecture. Adv Mater, 2017, 29: 1604572

8 Xu B R, Tian Z A, Wang J, et al. Stimuli-responsive and on-chip nanomembrane micro-rolls for enhanced macroscopic visual hydrogen detection. Sci Adv, 2018, 4: eaap8203

9 Tian Z A, Xu B R, Hsu B, et al. Reconfigurable vanadium dioxide nanomembranes and microtubes with controllable phase transition temperatures. Nano Lett, 2018, 18: 3017-3023

10 Hawkes E, An B, Benbernou N M, et al. Programmable matter by folding. Proc Natl Acad Sci USA, 2010, 107: 12441-12445 